\title{
ALMA update (Presentation Video)
}

\section{Pierre Cox}

Pierre Cox, "ALMA update (Presentation Video)," Proc. SPIE 9143, Space Telescopes and Instrumentation 2014: Optical, Infrared, and Millimeter Wave, 914311 (22 June 2014); doi: 10.1117/12.2063484

Event: SPIE Astronomical Telescopes + Instrumentation, 2014, Montréal, Quebec, Canada 


\title{
ALMA Update (Presentation Video)
}

\author{
Pierre Cox, Joint ALMA Observatory (Chile)
}

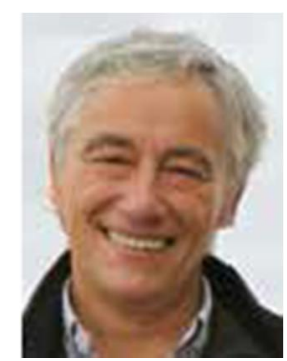

\begin{abstract}
The Atacama Large Millimeter/submillimeter Array is transitioning from construction to operations. This connected element array currently operates from wavelengths of 3-mm to 350-microns with up to 66 array elements, 54 of $12-\mathrm{m}$ diameter and 12 of 7-m diameter. While the antennas and most of the hardware for the receivers are on site, array capabilities are still expanding rapidly. In parallel with construction activities, early science observations have been going on since October 2011. At the time of the meeting, ALMA will be starting the third cycle of observing with many exciting, fundamental results already obtained. We will present the current status of the project and give an overview of the trailblazing science results obtained so far. The potential of the fully operational ALMA will be outlined as well as some of the development projects that are considered. In summary, this talk will address the past, present and future of ALMA, describe the transformational science that is and will be produced with ALMA.
\end{abstract}

View presentation video on SPIE's Digital Library: http://dx.doi.org/10.1117/12.2063484.3664756646001 